\title{
Surgical management of trigeminal schwannomas: defining the role for endoscopic endonasal approaches
}

\author{
ShaAn M. Raza, M.D., ${ }^{1}$ Angela M. Donaldson, M.D., ${ }^{2}$ Alpesh Mehta, M.D., ${ }^{4}$ \\ Apostolos J. Tsiouris, M.D., ${ }^{4}$ ViJay K. Anand, M.D., ${ }^{2}$ \\ AND ThEODORE H. SCHWARTZ, M.D. ${ }^{1-3}$ \\ Departments of ${ }^{1}$ Neurosurgery, ${ }^{2}$ Otolaryngology, ${ }^{3}$ Neuroscience, and ${ }^{4}$ Neuroradiology, Weill Cornell Medical \\ College, NewYork-Presbyterian Hospital, New York, New York
}

Object. Because multiple anatomical compartments are involved, the surgical management of trigeminal schwannomas requires a spectrum of cranial base approaches. The endoscopic endonasal approach to Meckel's cave provides a minimal access corridor for surgery, but few reports have assessed outcomes of the procedure or provided guidelines for case selection.

Methods. A prospectively acquired database of 680 endoscopic endonasal cases was queried for trigeminal schwannoma cases. Clinical charts, radiographic images, and long-term outcomes were reviewed to determine outcome and success in removing tumor from each compartment traversed by the trigeminal nerve.

Results. Four patients had undergone endoscopic resection of trigeminal schwannomas via the transpterygoid approach (mean follow-up 37 months). All patients had disease within Meckel's cave, and 1 patient had extension into the posterior fossa. Gross-total resection was achieved in 3 patients whose tumors were purely extracranial. One patient with combined Meckel's cave and posterior fossa tumor had complete resection of the extracranial disease and $52 \%$ resection of the posterior fossa disease. One patient with posterior fossa disease experienced a sixth cranial nerve palsy in addition to a corneal keratopathy from worsened trigeminal neuropathy. There were no CSF leaks. Over the course of the study, 1 patient with subtotal resection required subsequent stereotactic radiosurgery for disease progression within the posterior fossa.

Conclusions. Endoscopic endonasal approaches appear to be well suited for trigeminal schwannomas restricted to Meckel's cave and/or extracranial segments of the nerve. Lateral transcranial skull base approaches should be considered for patients with posterior fossa disease. Further multiinstitutional studies will be necessary for adequate power to help determine relative indications between endoscopic and transcranial skull base approaches. (http://thejns.org/doi/abs/10.3171/2014.7.FOCUS14341)

\section{KeY Words - trigeminal schwannoma - Meckel's cave • endoscopy • transpterygoid • skull base • minimally invasive}

$\mathrm{T}$ The most common skull base schwannoma after vestibular schwannomas, trigeminal schwannomas account for up to $0.36 \%$ of all intracranial neoplasms and $8 \%$ of skull base schwannomas. ${ }^{11,17,22}$ They are most often histologically benign lesions, and their anatomical characteristics can pose challenges to surgical management. While a majority of these tumors develop in the gasserian ganglion within the middle fossa, trigeminal schwannomas can occur anywhere along the course of the trigeminal root, ganglion, and peripheral branches. As a result, they can be intradural, interdural, and extradural and can exist in the posterior fossa, middle fossa/Meckel's cave, and extend along V1 into the orbit, V2 into the pterygopalatine fossa (PPF), and V3 into the infratemporal fossa. Given surrounding neuro-

Abbreviations used in this paper: $\mathrm{CN}=$ cranial nerve; $\mathrm{ICA}=$ internal carotid artery; PPF $=$ pterygopalatine fossa. vascular structures, trigeminal schwannomas can also be intimately associated with adjacent cranial nerves (CNs; for example, the abducens nerve) and vascular structures (that is, the horizontal petrous segment of the internal carotid artery [ICA]).

Given the higher recurrence rates with near-total resection as compared with those following gross-total resection, the goal of surgery is considered to be complete resection. Stereotactic radiosurgery has also been shown to be effective in controlling tumor growth. ${ }^{7}$ Numerous microsurgical approaches ranging from ventral (for example, transmaxillary), anterolateral (for example, orbitozygomatic), lateral (for example, subtemporal, preauricular infratemporal fossa), to posterolateral (for example, retrosigmoid suprameatal, presigmoid) have been reported in the surgical management of this disease entity.,3,17,21 In general, the reported rates of success from microsurgical removal have been favorable. Independent of location 
and surgical approach, gross-total resection rates as high as $82 \%$ have been reported. ${ }^{21}$ Functional outcomes have varied depending on the existence of preoperative symptoms, tumor size, and location. In larger reported series, either permanent or transient postoperative deterioration in neurological function, including trigeminal neuropathy, abducens nerve palsy, and trigeminal neuralgia, is experienced. These functional outcomes could be related to the anatomical disadvantage that characterizes laterally based skull base approaches. Hence, while the overall oncological and functional outcomes have improved with refinements in technique, the question of whether these outcomes can be improved arises.

Providing a ventral trajectory, endoscopic endonasal transpterygoid approaches to Meckel's cave have been described in several publications. ${ }^{8,10,16,20}$ Working through an anteromedial window into this region, endoscopic approaches provide the theoretical benefit of panoramic access. While several series have reported the feasibility of endoscopic approaches to Meckel's cave, there is no clear understanding of the relative advantages and disadvantages for this approach as regards the management of trigeminal schwannomas. In an effort to shed light on the endoscopic management of this pathology, we review our outcomes in this cohort of patients.

\section{Methods}

\section{Study Design}

After obtaining appropriate institutional review board approval for this study (Weil Cornell Medical CollegeNewYork-Presbyterian Hospital), we queried a prospectively acquired database of endoscopic endonasal skull base surgeries performed by the senior authors (T.H.S. and V.K.A.) at Weill Cornell Medical College, Sackler Brain and Spine Center, and NewYork-Presbyterian Hospital. We were interested in only those patients who had undergone pure endoscopic endonasal approaches for planned, single-stage gross-total resection of trigeminal schwannomas. Once patients were selected based on this criterion, we performed a retrospective chart review of hospital and office records for the remaining pre-, intra-, and postoperative treatment-related parameters. Particular attention was paid to pre- and postoperative $\mathrm{CN}$ deficits, including those related to $\mathrm{CN} \mathrm{V}$ (and its main branches), the vidian nerve, and the nerves responsible for extraocular muscle movement. Of note, for patients whose imaging studies were available, volumetric analysis was performed to determine the extent of resection for tumor components within the middle cranial fossa, posterior fossa, and extracranial compartments, depending on the extent of the preoperative disease burden.

\section{Surgical Technique}

We have described our endoscopic endonasal techniques and, in particular, the transpterygoid approach in detail elsewhere. ${ }^{8}$ Briefly, after intravenous premedication with dexamethasone $(10 \mathrm{mg})$ and diphenhydramine $(50 \mathrm{mg})$, patients receive $0.25 \mathrm{ml}$ of $10 \%$ intrathecal fluorescein (AK-Fluor, Akorn). Fluorescein is diluted with 10 $\mathrm{ml}$ of CSF withdrawn and administered over several minutes through either a lumbar puncture or a lumbar drain. The latter is generally used in patients in whom a highflow CSF leak is expected. After rigid fixation with the head slightly extended and rotated to the right, surgery is commenced. Visualization is provided with $0^{\circ}, 30^{\circ}$, and $45^{\circ}$ rigid 4-mm endoscopes, a digital camera, and a 21 -in color monitor (all three Karl Storz GmbH \& Co.). Using a rigid $0^{\circ}$ endoscope, we infiltrate the sphenopalatine foramen, uncinate process, vertical lamella of the ipsilateral middle turbinate, and bilateral mucoperichondrial septal flaps using 1\% lidocaine with 1:100,000 epinephrine to constrict branches of the sphenopalatine artery. A nasoseptal flap contralateral to the target lesion is harvested and stored in the nasopharynx. The flap's width is maximized by harvested tissue along the floor of the hard palate toward the inferior turbinate, whereas extending the harvesting incisions anteriorly to the columella ensures an adequate length for reconstruction. Bilateral flaps can also be harvested if additional coverage is needed. ${ }^{13}$

To fully expose the transmaxillary corridor, a complete ethmoidectomy and sphenoidotomy are performed first. The approach commences with an uncinectomy, a middle turbinate resection, and a wide maxillary antrostomy such that the infraorbital neurovascular bundle is visualized in the roof and the entire posterior maxillary sinus wall is exposed. An ethmoidectomy is then performed in a stepwise manner, which includes removal of the ethmoid bulla, suprabullar cells, and all the posterior ethmoid cells. The fovea ethmoidalis is identified and preserved. The sphenoid sinus is entered and widely enlarged. A wide sphenoid and ethmoid sinus cavity with identification of the lamina papyracea and skull base provides a panoramic view prior to exposing the PPF and pterygoid plates.

The sphenopalatine artery is then cauterized with an endoscopic bipolar probe and sectioned at its foramen. The vertical and orbital processes of the palatine bone are then removed using a combination of the endoscopic drill, Kerrison punches, and bone curettes. Removal of the posterior maxillary wall exposes the ventral periosteal sheath of the PPF. To fully expose the lateral sphenoid, the contents of the PPF are retracted laterally to identify the vidian nerve at the junction of the medial pterygoid plate and the floor of the sphenoid sinus. Meckel's cave is approached via a "supravidian" trajectory, which does not require sacrifice of this neurovascular bundle. The vidian nerve is exposed back to the junction of the paraclival and laceral segments of the ICA. Once this depth has been established, the pterygoid wedge (junction of the medial and lateral plates) is fully exposed. When viewed en face, toward the aspect of the pterygoid process, V2 can be found traversing the foramen rotundum into the PPF. Once this key landmark is noted, the pterygoid process can be drilled in a superior to inferior and medial to lateral fashion following V2 into the middle cranial fossa. This portion of the bony resection will provide access to the quadrangular space, which is bounded medially by the paraclival carotid and laterally by the maxillary nerve; the abducens nerve running along with V1 represents the superior limit of this space. The decision to 


\section{Endoscopic endonasal resection of trigeminal schwannomas}

expose the paraclival and laceral segments of the carotid artery depends on the access required for safe resection. We typically perform this exposure if vascular manipulation is required to gain access to tumor posterior to the ICA. These segments can be safely exposed using a combination of endoscopic drills and dissectors.

Entry into the infratemporal fossa or lateral aspect of the middle fossa is necessary for V3 involvement. Within the maxillary sinus, the infraorbital neurovascular bundle demarcates the PPF (medial) from the infratemporal fossa (lateral). After removing the posterior maxillary wall (described above), we follow the lateral pterygoid plate posteriorly to identify the mandibular nerve as it exits the foramen ovale. This stage requires a subperiosteal dissection to separate the inferior head of the lateral pterygoid muscle from the pterygoid plates to avoid frustrating venous bleeding from the pterygoid venous plexus. Once the V3 trunks have been identified, both the medial and lateral pterygoid plates are resected to gain the lateral access necessary for resection.

Once the main divisions of the trigeminal nerve and Meckel's cave have been identified, an incision is made in the periosteal dural layer to gain access to tumor within Meckel's cave. Tumor is subsequently resected using standard microsurgical instruments and a tissue aspirator as needed. Based on the growth of the tumor, the trigeminal nerve is typically displaced superolaterally toward the medial temporal lobe, while the abducens nerve lies along the superior aspect of the tumor. The best way to avoid injuring the abducens nerve is to not cross the superior aspect of $\mathrm{V} 2 .^{2}$

Skull base reconstruction is individualized for each patient depending on the extent of CSF leakage encountered. While our techniques for a multilayer closure and flap harvest have been described elsewhere, ${ }^{12,14}$ the nuances of closing laterally placed defects should be discussed. Resection of trigeminal schwannomas, particularly those extending into the posterior fossa, can result in large, laterally placed defects that may be challenging to access and reconstruct. As Meckel's cave exists within a separation of the two dural layers, oftentimes the meningeal dural layer is intact at the end of the resection, serving as a barrier against a high-flow CSF leak. As with all cranial base reconstructions, a multilayer closure represents the mainstay of our reconstruction. Most commonly, a fat graft is placed into the resection cavity, which is then covered with a nasoseptal flap harvested based on the contralateral sphenopalatine artery. It is imperative that the width and length of the flap are maximized during the initial portion of the operation to ensure that it will cover the cavity. Finally, although complete unroofing of the paraclival and petrous segments of the carotid artery is rare, the exposed vessel can be covered with fat or acellular debris bolstered with a tissue sealant. Limited lumbar drainage is used in cases of intraoperative high-volume CSF leaks for a period of 24-48 hours postoperatively to facilitate healing of this reconstruction.

\section{Results}

From a prospectively acquired database of 680 cases, we identified 4 patients who had undergone a purely endoscopic endonasal resection of trigeminal schwannoma. The cohort consisted of 2 males and 2 females ranging in age from 38 to 53 years, with a mean follow-up of 37 months (range 12-94 months). Varying degrees of trigeminal neuropathy involving one or two divisions were the most common form of presentation; two patients additionally presented with abducens nerve palsy (Table 1). With regard to the extent of disease presentation, one patient had involvement of Meckel's cave with extension into the posterior fossa, whereas the remaining patients had disease limited to Meckel's cave only. None of the cohort presented with extracranial disease extension.

All patients underwent resection via an endoscopic transpterygoid approach as described above. The posterior fossa was the only site where difficulty in performing a gross-total resection was experienced (Table 1); the only operative complications (palsy in CN VI and first division of $\mathrm{CN} \mathrm{V}$ ) occurred in this same case. The $\mathrm{CN}$ VI palsy was transient, improving 3 months postoperatively. No patient experienced a postoperative decrease in lacrimation from possible vidian nerve manipulation. Over a mean follow-up of 37 months, one incidence of progression was encountered 5 months postoperatively in the patient who had residual disease in the posterior fossa. This progression was treated with stereotactic radiosurgery. The remainder of the cohort had no evidence of radiographic disease on their most recent imaging studies. There were no CSF leaks.

\section{Illustrative Cases}

Note that a description of Case 4 is not included here since imaging studies and intraoperative images were not available.

\section{Case 1}

This 38-year-old man presented with left-sided V2/ V3 numbness with masseter weakness and mild CN VI palsy. His preoperative imaging studies (Fig. 1 upper) demonstrated a trigeminal schwannoma restricted to Meckel's cave. He was taken to the operating room for an endoscopic transpterygoid resection; intraoperative images are shown in Fig. 2 and Video 1.

VIDEo 1. Operative video for Case 1: a left-sided transpterygoid approach for a trigeminal schwannoma and a right-sided transpterygoid approach for a trigeminal schwannoma restricted to Meckel's cave, respectively. Copyright Theodore Schwartz. Published with permission. Click here to view with Media Player. Click here to view with Quicktime.

Postoperative imaging confirmed gross-total resection (Fig. 1 lower). The patient's preoperative $\mathrm{CN}$ deficits improved to full function by his 3-month postoperative visit.

\section{Case 2}

This 42-year-old man presented with left-sided V1/ V2 numbness and a hemisensory deficit. Preoperative imaging (Fig. 3 left) demonstrated a dumbbell-shaped trigeminal schwannoma involving both Meckel's cave and the posterior fossa. The patient underwent an endoscopic 
TABLE 1: Overview of outcomes from endoscopic transpterygoid resection of trigeminal schwannomas*

\begin{tabular}{|c|c|c|c|c|c|c|c|c|}
\hline $\begin{array}{l}\text { Age (yrs) } \\
\text { Sex }\end{array}$ & $\begin{array}{l}\text { Presenting } \\
\text { Symptom }\end{array}$ & $\begin{array}{l}\text { Disease w/ in } \\
\text { Posterior Fossa }\end{array}$ & $\begin{array}{l}\text { Extent of } \\
\text { Resection }\end{array}$ & $\begin{array}{l}\text { \% Tumor Vol } \\
\text { Remaining }\end{array}$ & Complication & Recurrence & $\begin{array}{l}\text { Disease Status } \\
\text { at Last FU }\end{array}$ & $\begin{array}{l}\text { Duration of } \\
\text { FU (mos) }\end{array}$ \\
\hline $38, M$ & $\begin{array}{l}\text { V2 numbness, } \mathrm{CN} \\
\quad \text { VI palsy }\end{array}$ & no & GTR & 0 & none & no & NED & 24 \\
\hline $40, M$ & V2 numbness & yes & STR & $52 \% \dagger$ & $\begin{array}{l}\text { CN VI palsy, V1 } \\
\text { numbness }\end{array}$ & $\begin{array}{l}\text { progression of posterior } \\
\text { fossa disease }\end{array}$ & stable disease & 19 \\
\hline $59, \mathrm{~F}$ & CN VI palsy & no & GTR & 0 & none & no & NED & 94 \\
\hline $53, F$ & V2/V3 numbness & no & GTR & 0 & none & no & NED & 12 \\
\hline
\end{tabular}

* FU = follow-up; GTR = gross-total resection; NED = no evidence of disease; STR = subtotal resection.

$\dagger$ Posterior fossa component.
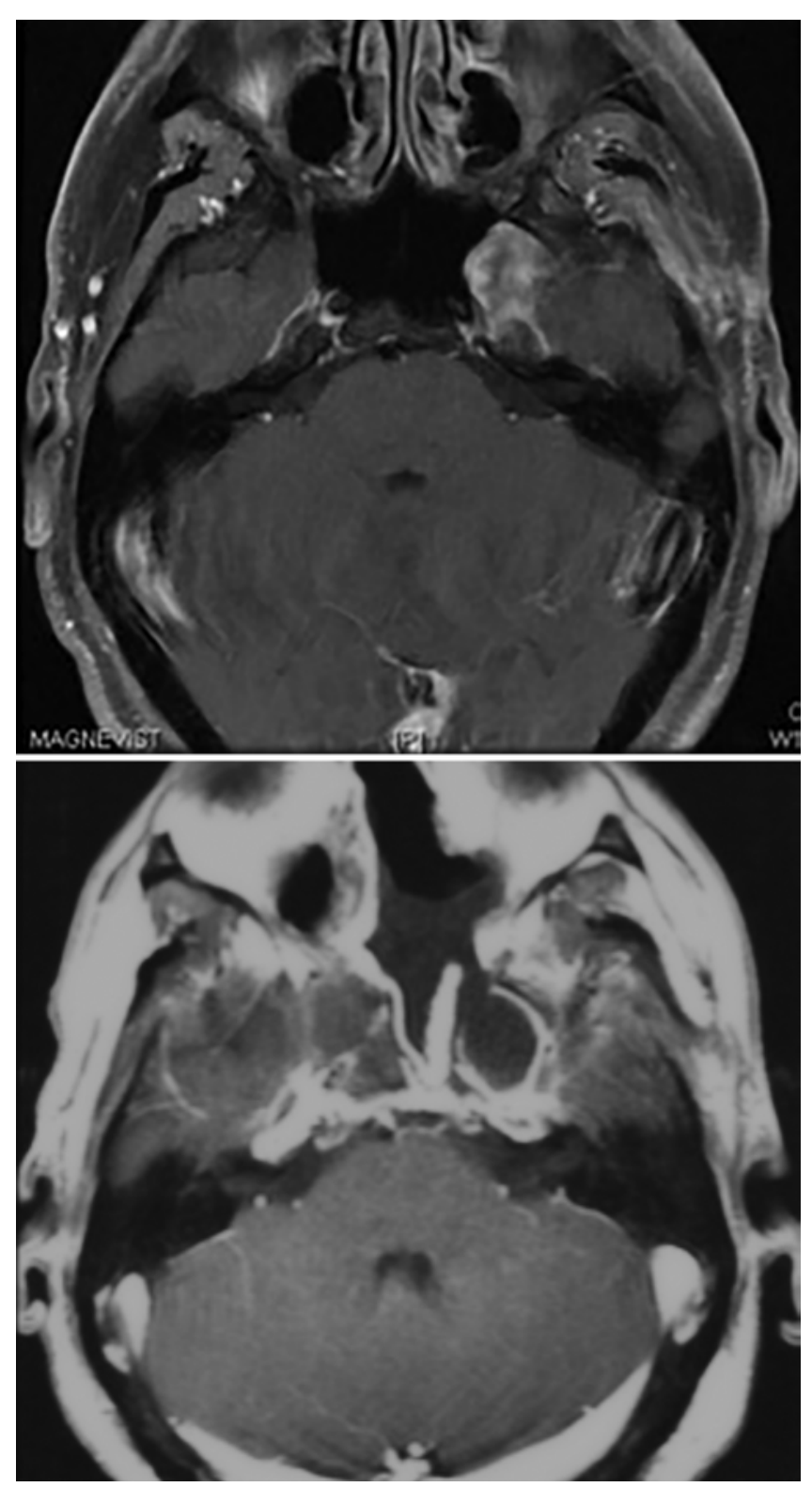

FIG. 1. Case 1. Upper: Preoperative T1-weighted MR image with contrast demonstrating an enhancing mass restricted to Meckel's cave. Lower: Postoperative T1-weighted MR image with contrast confirming gross-total resection. transpterygoid resection. Postoperative imaging (Fig. 3 right) demonstrated subtotal resection with residual disease located in the cerebellopontine cistern within the posterior fossa. Nevertheless, the brainstem was decompressed and the hemisensory deficit was resolved. The patient suffered a transient CN VI palsy in addition to a corneal ulcer due to worsened neuropathy in the first division of $\mathrm{CN}$ V.

\section{Case 3}

This 53-year-old woman presented with right-sided masseter weakness in addition to V3 distribution numbness. Preoperative imaging (Fig. 4 upper) demonstrated a mass within Meckel's cave. The patient underwent an endoscopic transpterygoid resection. Postoperative imaging demonstrated gross-total resection (Fig. 4 lower). The patient suffered no postoperative complications and eventually recovered from her preoperative trigeminal neuropathy.

\section{Discussion}

Despite their low incidence, it is evident that, with rare exceptions, trigeminal schwannomas are benign lesions for which total resection can offer a durable cure, whereas subtotal resection is linked to an increased risk of recurrence. ${ }^{4,15,19}$ Despite their biologically benign behavior, these tumors can pose significant surgical challenges given their different sites of origin and extension along the main root, ganglion, and major divisions of the trigeminal nerve. Additionally, because of the neurovascular density of the middle and posterior fossae, target lesions can be intimately associated with adjacent structures such as the petrous ICA or brainstem. Several classification systems have been proposed as a foundation for a systematic approach in selecting the optimal surgical strategy. ${ }_{5,6,11,21,22}$ The most apt system is one proposed by Wanibuchi et al. based on 4 anatomical categories of tumor: peripheral, ganglion, root, and dumbbell. ${ }^{21}$ The peripheral type refers to tumor along V1 in the orbit, V2 in the PPF and maxilla, and V3 into the infratemporal fossa. The ganglion type primarily lies within the middle cranial fossa, whereas the root type lies along the cisternal segment of the trigeminal nerve in the brainstem. The dumbbell category of schwannoma can involve the ganglion and peripheral branches or the ganglion and posterior fossa. 


\section{Endoscopic endonasal resection of trigeminal schwannomas}

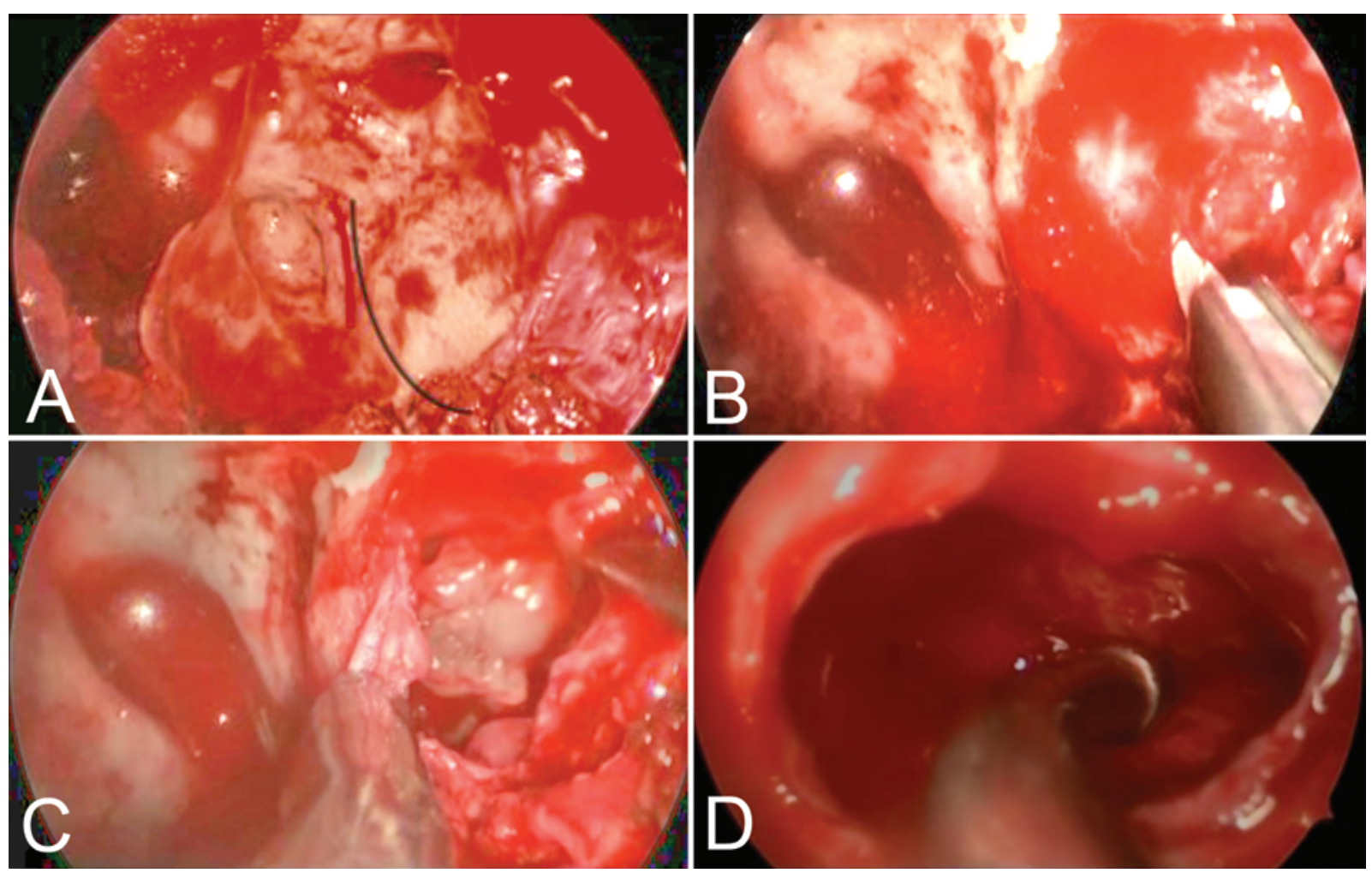

Fig. 2. Case 1. A: View obtained after sphenoidotomy, maxillary antrostomy, opening, and transposition of the PPF contents were performed. Note the expanded appearance of Meckel's cave because of the tumor. Paraclival ICA (solid red line, parallel to solid black line) and medial and inferior borders of Meckel's cave (solid black line). B: After removing the ventral and medial bone overlying Meckel's cave, we incised the periosteal dura to gain access to tumor. C: The tumor was internally debulked. D: After resection of the tumor capsule, the gasserian ganglion is visible.

Numerous surgical strategies based on tumor category have been reported by Wanibuchi et al., ${ }^{21}$ Al-Mefty et al., ${ }^{1}$ Samii et al., ${ }^{17}$ and Goel et al. ${ }^{5}$ in the 4 largest series to date. Unfortunately, given differences in the classification systems used, lack of volumetric measurements, and inconsistency in reporting postoperative $\mathrm{CN}$ function, it is difficult to combine data to draw conclusions regarding the efficacy of lateral skull base approaches. An overview of the results is shown in Table 2. With the full spectrum of tumor classifications represented among the cohorts, gross-total resection rates of up to $83 \%$ were reported, with complication rates ranging between $1.9 \%$ and $8 \%$. Unfortunately, according to the reports, it is difficult to
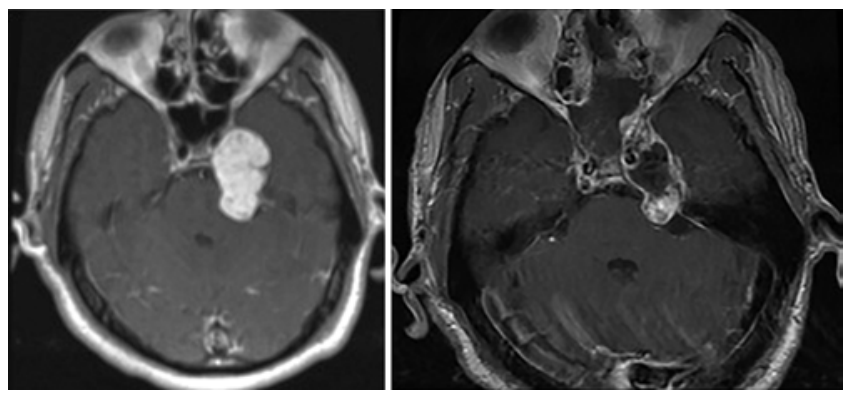

FIG. 3. Case 2. Left: Preoperative T1-weighted MR image with contrast demonstrating an enhancing mass within both Meckel's cave and the posterior fossa. Right: Postoperative T1-weighted MR image with contrast demonstrating residual disease in the posterior fossa. assess risk factors for subtotal resection via lateral skull base approaches. In their analysis, Wanibuchi et al. found that the total removal of large, solid dumbbell-type tumors tends to be difficult. ${ }^{21}$ In an analysis of subtotal or partial resections, these authors did not differentiate between middle-posterior fossa and middle-peripheral type tumors among the dumbbell-type tumors. Furthermore, those tumors intraoperatively considered to be highly vascular, fibrous, and adherent to surrounding neurovascular structures were more likely to be subtotally resected. However, tumors that cannot be completely resected may be amenable to stereotactic radiosurgery, which has been shown to provide 5-year progression-free survival of $95 \%$ in selected patients.?

As regards iatrogenic $\mathrm{CN}$ injury, the trigeminal nerve and its branches along with CNs within the cavernous sinus were at highest risk in the lateral approaches. While many patients had preoperative trigeminal neuropathy, rates of $11.6 \%-33 \%$ of worsened function in the form of facial pain, hypesthesia, or motor root dysfunction have been reported. While the operative risk of injury is probably related to tumor size and preoperative dysfunction, the concern with lateral skull base approaches is the extent of nerve manipulation required to dissect tumor from the inferomedial surface along the various segments of the nerve. Additionally, surgical injury to the abducens nerve $(8 \%)$, trochlear nerve $(3.7 \%)$, and oculomotor nerve $(1.9 \%)$ has been reported. The relative rates of injury to 

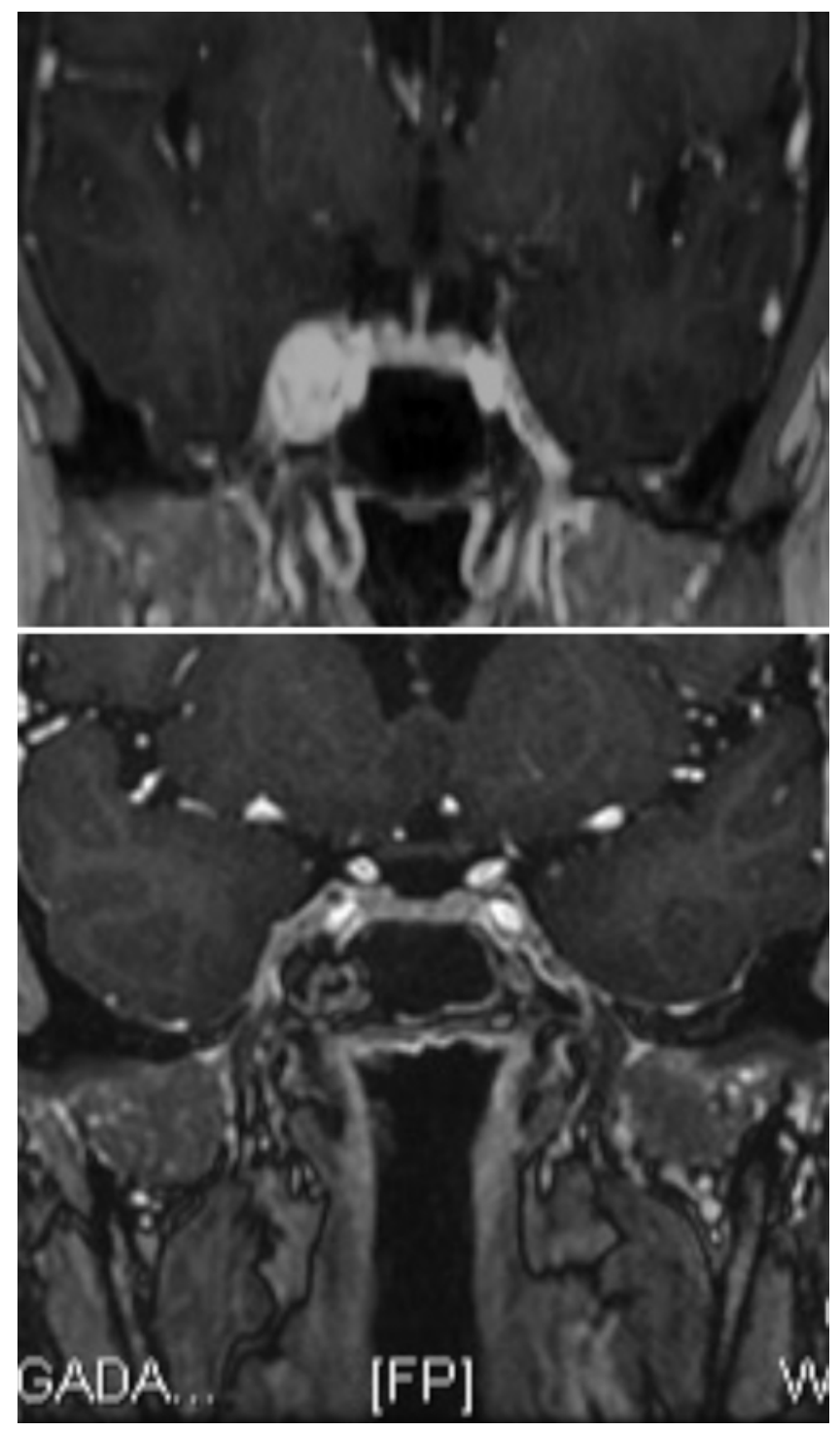

FIG. 4. Case 3. Upper: Preoperative T1-weighted MR image with contrast demonstrating an enhancing mass within Meckel's cave. Lower: Postoperative T1-weighted MR image with contrast demonstrating gross-total resection.

the trochlear nerve and oculomotor nerve indicate that the size of the middle fossa-cavernous sinus tumor is the likely risk factor given that the trochlear nerve sits closer to V1 than the oculomotor nerve.

The complicated anatomical course of the abducens nerve places the nerve at varying degrees of risk depending on the category and size of tumor as well as the approach selected. The nerve's path can be divided into the following segments: cisternal, petrosal, cavernous, and intraorbital. ${ }^{2}$ Within the posterior fossa, as the cisternal segment of CN VI exits the pontomedullary sulcus at the level of the vertebrobasilar junction and travels toward Dorello's canal, the nerve lies inferior and medial to the trigeminal nerve. At the level of the petroclinoid ligament (Gruber's ligament), the petrosal segment of the nerve enters Dorello's canal directly medial to the trigeminal nerve root. As the nerve transitions to its cavernous seg- ment, the nerve takes a slightly superior trajectory to the trigeminal nerve and eventually lies within the lateral compartment of the cavernous sinus medial and superior to V1 (the superior-most branch of the trigeminal nerve within the lateral cavernous sinus wall). As regards $\mathrm{CN}$ VI preservation, it is feasible that the lateral skull base approaches are well suited for middle-posterior fossa tumors approached prior to the nerve and for which sharp dissection can be performed when encountered. However, toward the cavernous sinus and anteriorly, CN VI transitions to a higher pathway where it is never really directly dissected via a transcranial approach unless the supratrochlear triangle in the lateral cavernous sinus wall is opened-placing CN IV at unnecessary risk. ${ }^{9}$ At this segment of CN IV, more medially based approaches would provide direct visualization.

Endoscopic endonasal transpterygoid approaches have been proposed as a viable option in accessing lesions within Meckel's cave..$^{8,10,18,20}$ Numerous reports have documented the management of a spectrum of middle fossa pathologies in this region-ranging from encephaloceles to perineural spread of adenoid cystic carcinoma. With the transposition of the PPF contents and a supravidian approach, V2 can be identified at the foramen rotundum toward the superior aspect of the pterygoid wedge and followed back to the gasserian ganglion. With resection of the pterygoid plates, V3 and its two trunks can be identified in the pterygomaxillary space of the infratemporal fossa and followed to the foramen ovale. The provided ventromedial approach places the trigeminal nerve at the lateral extent of the exposure, while the abducens nerve lies toward the superior aspect of the field. Specifically, as regards trigeminal schwannomas, the theoretical benefit would be reduced manipulation of the trigeminal nerve, translating into reduced rates of postoperative trigeminal neuropathy. Additionally, CN VI could be directly visualized, facilitating extracapsular dissection away from the nerve.

Because of the limited number of cases performed at any one center, a paucity of data directly address the role of endoscopic endonasal approaches for trigeminal schwannomas. Shin et al., in their series of endoscopically resected nonvestibular schwannomas, had 11 trigeminal schwannomas. ${ }^{18}$ Among this cohort, 4 patients had primarily middle fossa disease, 2 had combined middleposterior fossa tumor, 3 had combined middle-peripheral disease, and 2 presented with isolated extracranial disease. Those patients with disease extending into the posterior fossa underwent staged resections combined with a retrosigmoid approach. In this reported series, the rate of gross-total resection was $63 \%$ with a postoperative trigeminal neuropathy (motor and/or sensory) rate of $45 \%$. Additionally, 54\% of patients reported dry eye postresection, and $9 \%$ experienced abducens nerve palsy.

When our study and Shin et al.'s are placed in the context of published reports of lateral skull base approaches, it appears that endoscopic approaches provide adequate access, as supported by the rates of gross-total resection. However, a ventral approach may have limited utility for posterior fossa disease given that the only subtotal resection we described was for a combined middle-posterior 
Endoscopic endonasal resection of trigeminal schwannomas

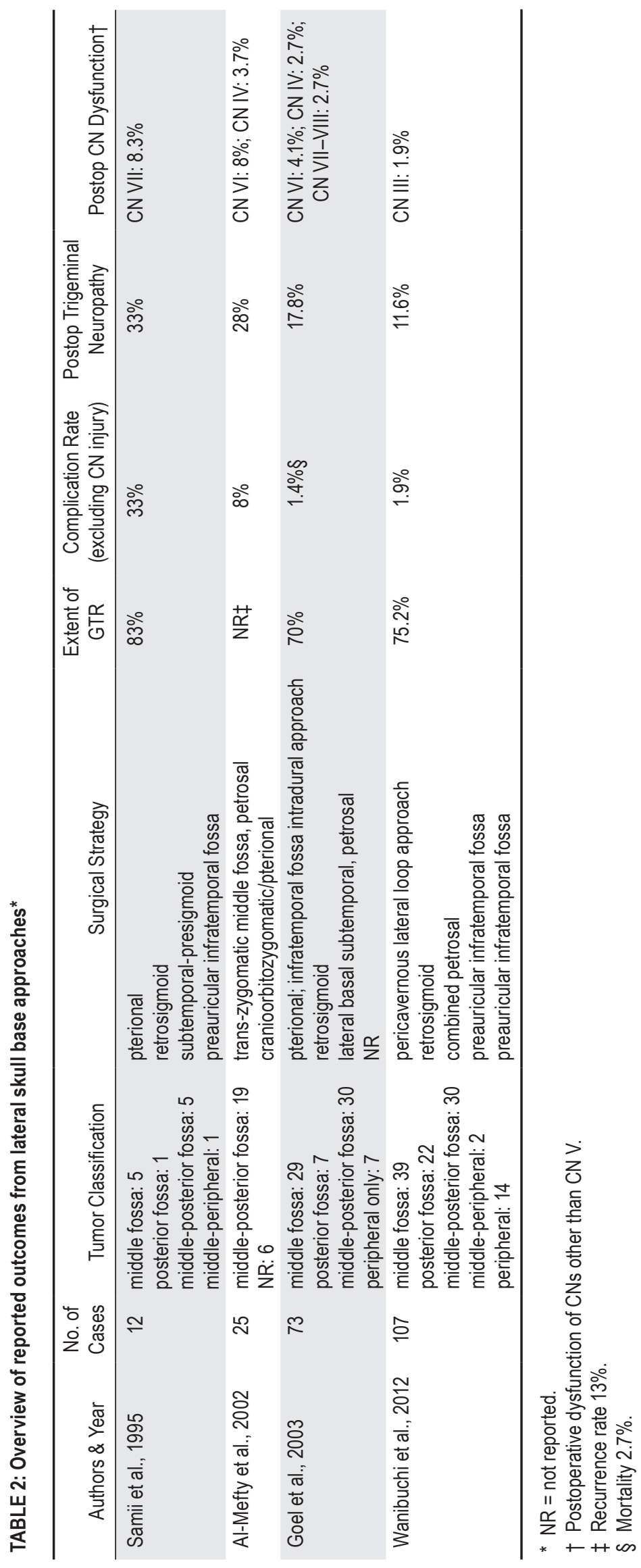


fossa tumor, whereas Shin et al. required staged excisions to achieve a resection in this category. While the porus trigeminus can be opened further via a lateral skull base approach by sectioning the superior petrosal sinus, an expanded endonasal approach into the posterior fossa at this level would require skeletonization of the paraclival and laceral segments of the ICA as well as, perhaps, an infrapetrous approach. The limitation of the endoscopic approach in accessing posterior fossa disease is supported by the higher rate of CN VI palsy in this tumor category. Otherwise, according to the data, endoscopic transpterygoid approaches provide safe access to Meckel's cave and disease extending along V2 into the PPF and V3 into the infratemporal fossa without risking additional $\mathrm{CN}$ morbidity. Alternatively, stereotactic radiosurgery can be used to control residual disease in the posterior fossa if decompression of the brainstem is not required.

The reported rates of dry eye and corneal neurotrophic keratopathy require consideration given that either can be a devastating complication. The presumed mechanism of these complications would be attributable to either vidian nerve injury (if the vidian is transected) or a V1 neuropathy. In our and Shin et al.'s studies, corneal keratopathy was seen in patients with both V1 neuropathy and complaints of dry eye. While an endoscopic transpterygoid approach to Meckel's cave is typically supravidian and does not require the nerve's sacrifice, transposition of the PPF contents could theoretically result in vidian nerve injury. Given that an endoscopic approach directly places this nerve at risk, the serious threat of corneal keratopathy should be considered in those patients with either preoperative V1 neuropathy or tumor extending along this division.

\section{Conclusions}

Endoscopic endonasal approaches are growing in popularity in the management of skull base pathology. Guidelines for the application of endonasal surgery for resecting trigeminal schwannomas are lacking. Our results suggest that endoscopic transpterygoid approaches can help to achieve optimal resection rates with limited $\mathrm{CN}$ morbidity for tumors isolated to Meckel's cave, with combined Meckel's cave-peripheral extension, or primarily extracranial in location. Thus far, outcomes in the management of tumors with posterior fossa extension in patients with preoperative V1 neuropathy have been less than ideal. Unfortunately, because of the small number of patients in our study and described in the literature, it is difficult to ascertain the utility of endoscopic endonasal approaches in the management of trigeminal schwannomas. This is compounded by the low incidence and anatomical variability of these tumors. Future multiinstitutional studies with standardized outcome assessment will be necessary to determine the ideal surgical approach for each category of trigeminal schwannoma.

\section{Disclosure}

The authors report no conflict of interest concerning the materials or methods used in this study or the findings specified in this paper. Dr. Schwartz is a consultant for Karl Storz, has direct stock ownership in Visionsense, and has received support from the NIH for non-study-related clinical or research effort.

Author contributions to the study and manuscript preparation include the following. Conception and design: Schwartz, Raza. Acquisition of data: Raza, Mehta. Analysis and interpretation of data: Schwartz, Raza, Donaldson, Tsiouris. Drafting the article: Schwartz, Raza, Donaldson. Critically revising the article: Schwartz, Raza, Donaldson, Anand. Reviewed submitted version of manuscript: Schwartz, Raza. Approved the final version of the manuscript on behalf of all authors: Schwartz. Administrative/technical/material support: Schwartz. Study supervision: Schwartz.

\section{References}

1. Al-Mefty O, Ayoubi S, Gaber E: Trigeminal schwannomas: removal of dumbbell-shaped tumors through the expanded Meckel cave and outcomes of cranial nerve function. J Neurosurg 96:453-463, 2002

2. Barges-Coll J, Fernandez-Miranda JC, Prevedello DM, Gardner P, Morera V, Madhok R, et al: Avoiding injury to the abducens nerve during expanded endonasal endoscopic surgery: anatomic and clinical case studies. Neurosurgery 67:144154,2010

3. Day JD, Fukushima T: The surgical management of trigeminal neuromas. Neurosurgery 42:233-241, 1998

4. Dolenc VV: Frontotemporal epidural approach to trigeminal neurinomas. Acta Neurochir (Wien) 130:55-65, 1994

5. Goel A, Muzumdar D, Raman C: Trigeminal neuroma: analysis of surgical experience with 73 cases. Neurosurgery 52: 783-790, 2003

6. Guthikonda B, Theodosopoulos PV, van Loveren H, Tew JM $\mathrm{Jr}$, Pensak ML: Evolution in the assessment and management of trigeminal schwannoma. Laryngoscope 118:195-203, 2008

7. Hasegawa T, Kato T, Iizuka H, Kida Y: Long-term results for trigeminal schwannomas treated with gamma knife surgery. Int J Radiat Oncol Biol Phys 87:1115-1121, 2013

8. Hofstetter CP, Singh A, Anand VK, Kacker A, Schwartz TH: The endoscopic, endonasal, transmaxillary transpterygoid approach to the pterygopalatine fossa, infratemporal fossa, petrous apex, and the Meckel cave. Clinical article. J Neurosurg 113:967-974, 2010

9. Isolan GR, Krayenbühl N, de Oliveira E, Al-Mefty O: Microsurgical anatomy of the cavernous sinus: measurements of the triangles in and around it. Skull Base 17:357-367, 2007

10. Kassam AB, Prevedello DM, Carrau RL, Snyderman CH, Gardner P, Osawa S, et al: The front door to Meckel's cave: an anteromedial corridor via expanded endoscopic endonasal approach-technical considerations and clinical series. Neurosurgery 64 (3 Suppl):ons71-ons83, 2009

11. Lesoin F, Rousseaux M, Villette L, Autricque A, Dhellemmes P, Pellerin P, et al: Neurinomas of the trigeminal nerve. Acta Neurochir (Wien) 82:118-122, 1986

12. McCoul ED, Anand VK, Singh A, Nyquist GG, Schaberg MR, Schwartz TH: Long-term effectiveness of a reconstructive protocol using the nasoseptal flap after endoscopic skull base surgery. World Neurosurg 81:136-143, 2014

13. Nyquist GG, Anand VK, Singh A, Schwartz TH: Janus flap: bilateral nasoseptal flaps for anterior skull base reconstruction. Otolaryngol Head Neck Surg 142:327-331, 2010

14. Patel KS, Komotar RJ, Szentirmai O, Moussazadeh N, Raper DM, Starke RM, et al: Case-specific protocol to reduce cerebrospinal fluid leakage after endonasal endoscopic surgery. Clinical article. J Neurosurg 119:661-668, 2013

15. Pollack IF, Sekhar LN, Jannetta PJ, Janecka IP: Neurilemomas of the trigeminal nerve. J Neurosurg 70:737-745, 1989

16. Roth J, Singh A, Nyquist G, Fraser JF, Bernardo A, Anand VK, et al: Three-dimensional and 2-dimensional endoscopic exposure of midline cranial base targets using expanded en- 


\section{Endoscopic endonasal resection of trigeminal schwannomas}

donasal and transcranial approaches. Neurosurgery 65:11161130,2009

17. Samii M, Migliori MM, Tatagiba M, Babu R: Surgical treatment of trigeminal schwannomas. J Neurosurg 82:711-718, 1995

18. Shin SS, Gardner PA, Stefko ST, Madhok R, Fernandez-Miranda JC, Snyderman CH: Endoscopic endonasal approach for nonvestibular schwannomas. Neurosurgery 69:1046-1057, 2011

19. Taha JM, Tew JM Jr, van Loveren HR, Keller JT, el-Kalliny $\mathrm{M}$ : Comparison of conventional and skull base surgical approaches for the excision of trigeminal neurinomas. J Neurosurg 82:719-725, 1995

20. Van Rompaey J, Suruliraj A, Carrau R, Panizza B, Solares CA: Meckel's cave access: anatomic study comparing the endoscopic transantral and endonasal approaches. Eur Arch Otorhinolaryngol 271:787-794, 2014

21. Wanibuchi M, Fukushima T, Zomordi AR, Nonaka Y, Friedman AH: Trigeminal schwannomas: skull base approaches and operative results in 105 patients. Neurosurgery 70 (1 Suppl Operative):132-144, 2012
22. Yoshida K, Kawase T: Trigeminal neurinomas extending into multiple fossae: surgical methods and review of the literature. J Neurosurg 91:202-211, 1999

Manuscript submitted June 15, 2014.

Accepted July 30, 2014.

Please include this information when citing this paper: DOI: 10.3171/2014.7.FOCUS14341.

Supplemental online information:

Video 1: http://mfile.akamai.com/21490/wmv/digitalwbc.download. akamai.com/21492/wm.digitalsource-na-regional/focus14-341_ video_1.asx (Media Player).

http://mfile.akamai.com/21488/mov/digitalwbc.download.akamai. com/21492/qt.digitalsource-global/focus14-341_video_1.mov (Quicktime).

Address correspondence to: Theodore H. Schwarz, M.D., Departments of Neurosurgery, Otolaryngology and Neuroscience, Weill Cornell Medical College, NewYork-Presbyterian Hospital, 525 E. 68th St., Box 99, New York, NY 10065. email: schwarh@cornell. med.edu. 